\title{
Left- sided Acute Appendicitis with Intestinal Malrotation in an Adult:a Diagnostic Challenge
}

\author{
Gautam R ${ }^{1}$, De Lima Gemma U ${ }^{2}$, Adhikari B $^{3}$ \\ Chitwan Medical College, Bharatpur, Nepal ${ }^{1}$, St. Luke's Medical Center Global City, \\ Philippines ${ }^{2}$, and Nepal Medical College, Kathmandu, Nepal ${ }^{3}$
}

Received: January 15, 2016

Accepted: May 20, 2016

Cite this paper: Gautam $\mathrm{R}^{1}$, De Lima Gemma $\mathrm{U}^{2}$, Adhikari B. Left- sided Acute Appendicitis with Intestinal Malrotation in an Adult:a Diagnostic Challenge. Nepalese Journal of Radiology 2017;7(10):27-30.

\begin{abstract}
A 28-yr-old male presented with sudden onset pain at the epigastric region followed by left lower abdominal pain. CT scan of the abdomen revealed reverse relation of the superior mesenteric artery and superior mesenteric vein with large bowel loops including the caecum on left side and small bowel loops on the right. An inflamed appendix arising from the base of left sided caecum was identified. The intra operative findings were consistent with CT features. This is a rare case of left sided acute appendicitis with intestinal malrotation in an adult patient. CT scan is diagnostic modality of choice and excludes other possible disease presenting with similar clinical features.
\end{abstract}

Keywords: Acute appendicitis, Adult presentation, Intestinal Malrotation

\section{INTRODUCTION}

Intestinal malrotation in an adult is uncommon. In addition malrotation presenting as acute left sided appendicitis is even rare with few individual case reports described in the literature. ${ }^{1,2}$ The unusual clinical presentation of left lower abdominal pain without bowel and urinary symptoms should arise the possibility of this rare clinical entity. Contrast enhanced CT-scan of the abdomen and pelvis is the diagnostic method of choice which reveals the anatomic details of intestinal malrotation, findings of acute appendiceal inflammation and its possible complications. Further imaging also helps to exclude other

Correspondence to: Dr. Rupesh Gautam, Chitwan Medical college, Bharatpur, Nepal Email:rupesh_gautam@hotmail.com disease, presenting with similar clinical features of acute left lower abdominal pain. 


\section{CASE REPORT}

A 28- yr-male presented to the emergency room with sudden onset of epigastric pain followed by left lower abdominal pain for last two hours. No abnormal urinary or bowel symptoms were noted, except for four episodes of non-projectile vomiting. No significant past medical or surgical illness was elicited. The patient was afebrile with normal blood pressure and heart rate. Physical examination revealed mild tenderness at both epigastric region and left lower abdomen.The only significant laboratory finding was elevated white blood cell count of 18,360 cells $/ \mathrm{mm}^{3}$ with $85 \%$ neutrophils (normal range 4,800 - 10,800 cells $/ \mathrm{mm} 3$ ). Urinalysis was within normal range. Further diagnostic workup

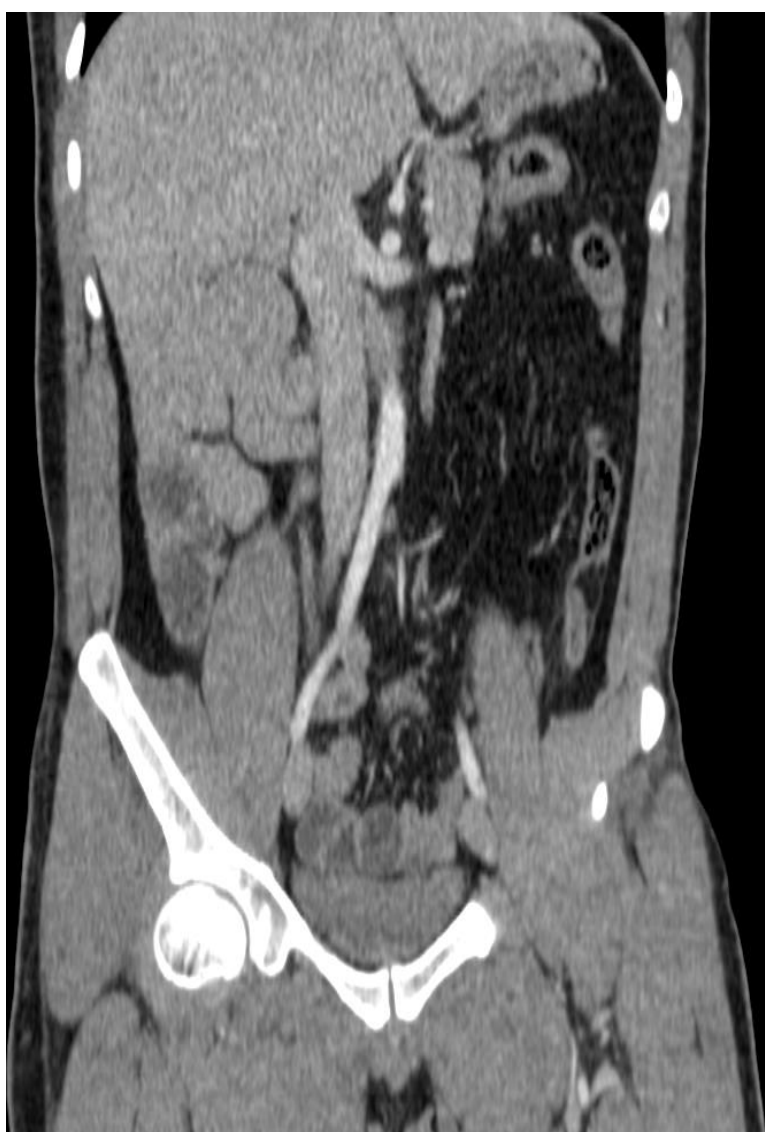

was done with intravenous non-ionic contrast enhanced CT scan of the abdomen and pelvis and revealed a blind ended tubular structure with maximal luminal diameter of $1.2 \mathrm{~cm}$ at the left lower abdomen arising from the left sided caecum (Fig.1 and 3.) The small bowel loops were located on the right side, whereas the large bowel loops were on the left(Fig.2). Likewise, the superior mesenteric artery was noted at the right of superior mesenteric vein, reversed relation (Fig.2), indicating Stringers Type I intestinal malrotation ${ }^{1}$. Normally the superior mesenteric vein lies right to the superior mesenteric artery ${ }^{3}$. The radiological diagnosis of left sided acute appendicitis with intestinal malrotation was made. Emergency laparatomy was done and intra operative findings were consistent with the CT-scan features.

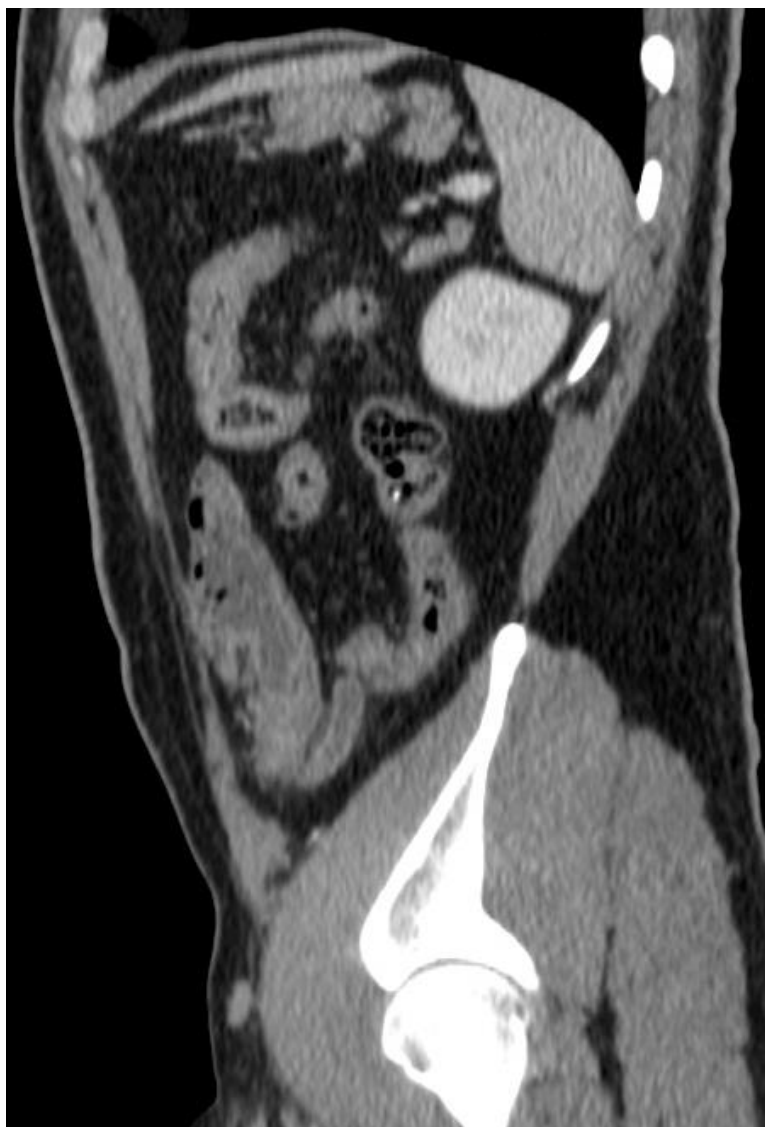

Figure 1 a and 1b: Coronal and Sagittal contrast enhanced CT scan of the abdomen demonstrates tubular structure at the left lower abdomen with surrounding fat stranding densities and haziness. This structure arises from the left sided caecum, confirming the findings of acute left appendicitis with intestinal malrotation. 


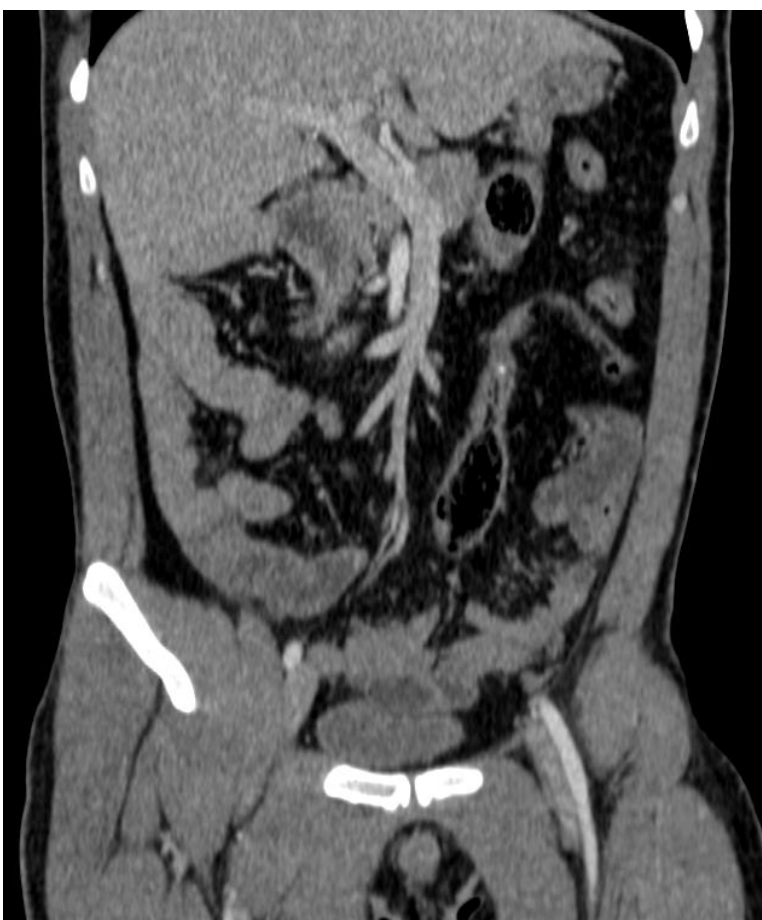

Figure 2: Coronal contrast enhanced CT scan of the abdomen demonstrates the inverse relation of superior mesenteric artery and vein. The smallbowel loops are located on the right and large bowel loops are on the left, all suggestive of Intestinal malrotation.

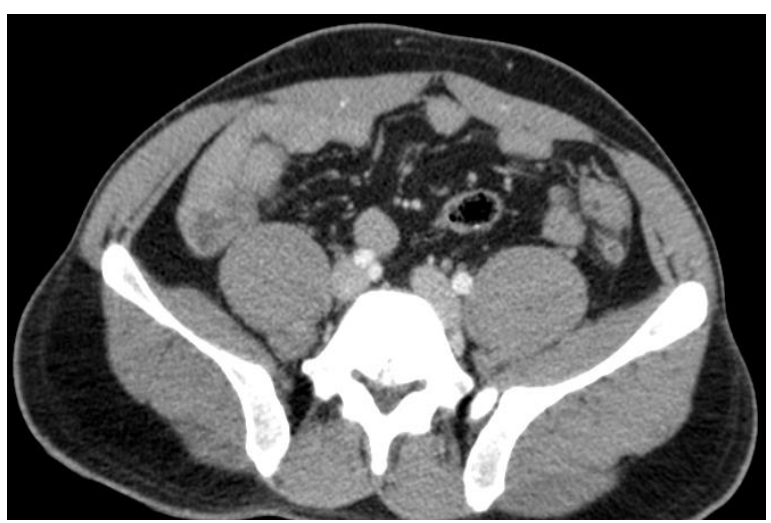

Figure 3: Axial contrast enhanced CT scan of the abdomen demonstrates tubular structure at the left lower abdomen with surrounding fat stranding densities and haziness.

\section{DISCUSSION}

Acute appendicitis is the major cause of visit to emergency room and perhaps the most common operation performed throughout the world. The incidence of acute appendicitis is 1.33 per 1000 in males and 0.99 per 1000 in females. ${ }^{2}$ Shifting abdominal pain from epigastric region to the right lower abdomen with tenderness oncompression are the classic clinical manifestations and usually associated with fever and vomiting. The appendix is normally less than $6 \mathrm{~mm}$ in diameter. The typical CT findings of uncomplicated acute appendicitis are dilatation of the appendix with diameter greater than $6 \mathrm{~mm}$, wall thickening and enhancement and peri-appendiceal inflammatory changes. The combination of clinical features and imaging findings suggest the diagnosis of acute appendicitis. ${ }^{3}$ Left sided appendicitis is rare and the possibilities include an abnormally enlarged right sided appendix with the tipat the left or appendicitis associated with intestinal malrotation or situs inversus totalis. Embryonic development of the gastrointestinal tract starts at 4th week of gestation. The total of 270 degrees anticlockwise rotation of the mid gut occurs until 12th week of gestation before it is fixed to the mesentery and posterior abdominal wall. ${ }^{4}$ It is during this time of development various forms of rotational anomalies take place.

Springer classified rotational abnormalities of the mid gut into three broad types as nonrotation, partial malrotation and reverse rotation. Non-rotation is the most common form of malrotation, where entire large bowel lies on the left hemi abdomen and duodenal junction lies to the right. The incidence of malrotation is 1 in 500 new born and 64$80 \%$ of the neonates present within first month of life. Upper gastrointestinal series is the investigation of choice for diagnosis of malrotation in children and demonstrates failure of the duodenal-jejunal junction to cross the midline and lies below duodenal bulb. ${ }^{5,6}$ Intestinal malrotation in adult is uncommon and occasionally noted asincidental finding on adult population. There are no large studies published for describing the incidence of intestinal malrotation in adult population. 
The common etiologies of left lower abdominal pain are ureteric colic, acute diverticulitis, inflammatory boweldisease, epiploic appendagitis, bowel ischemia, omental infarction, acute pancreatitis, ruptured ovarian cyst, epididymitis, bowel obstruction and psoas abscess. Intestinal malrotation in an adult patient presenting with acute left sided appendicitis is extremely rare with few individual case reports described in the literature and not commonly included as a differential diagnosis for left lower abdominal pain.

\section{CONCLUSION}

Left sided acute appendicitis is a rare clinical condition. Imaging plays a crucial role in timely diagnosis of this condition and appropriate management to prevent fatal complications. Contrast enhanced CT scan of the abdomen and pelvis is the diagnostic modality of choice.

\section{CONFLICT OF INTEREST}

None

\section{SOURCES OF FUNDING}

None

\section{REFERENCES}

1. Shekhar A, Hendahewa R, Premaratne G. A diagnostic dilemma: Leftsided appendicitis in a 10 year old boy with previously undiagnosed intestinal malrotation. Int $J$ Surg Case Rep 2015; 14:10-12. https://doi.org/10.1016/j. ijscr.2015.06.036

2. AkbulutS, UlkuA, SenolA, Tas M, Yagmur Y, Left-sided appendicitis: Review of 95 published cases and a case report, World $J$ Gastroenterol 2010; 16 (44): 5598-5602. https://doi.org/10.3748/wjg.v16. i44.5598
3. Nichols DM and Li DK, Superior mesenteric vein rotation: a CT sign of midgut malrotation, $A J R A m ~ J$ Roentgenol 1983; 141:707-708. https://doi.org/10.2214/ajr.141.4.707

4. Leite NP, Pereira JM, Cunha R, Pinto P, SirlinC.CTEvaluationofAppendicitisand Its Complications: Imaging Techniques and Key Diagnostic Findings. AJR Am J Roentgenol 2005; 185:406-417. https://doi.org/10.2214/ ajr.185.2.01850406

5. Pickhardt PJ, Bhalla S. Intestinal Malrotation in Adolescents and Adults: Spectrum of Clinical and Imaging Features. AJR Am J Roentgenol 2002;179:1429-1435. https://doi.org/10.2214/ ajr.179.6.1791429

6. Long FR, Kramer SS, Markowitz RI, Taylor GE. Radiographic Patterns of Intestinal Malrotation in children. RadioGraphics 1996; 16:547-556. https://doi.org/10.1148/ radiographics.16.3.8897623 